\title{
Trois ans de banque de données de la valeur intrinsèque: rétrospective et perspectives
}

\author{
Olivier Kappeler ${ }^{a}$ \\ Erika Flückiger ${ }^{\text {, }}$ \\ Karl Bachofenc, \\ Christoph Hänggeli ${ }^{d}$ \\ Jürg Jau ${ }^{e}$
}

a Membre du Comité central de la FMH

b Responsable des Services

c Economiste, Service tarifaire

d lic. en droit, administrateur du Secrétariat pour la formation prégraduée, postgraduée et continue

e Responsable TIC
1 Les médecins qui n'exercent pas sous leur propre responsabilité et ceux en formation postgraduée bénéficient de la garantie des droits acquis s'ils ont accompli sous supervision, pendant les 2 ans précédant l'introduction du TARMED, les prestations qu'ils souhaitent facturer, et ce, de manière régulière et sans contestation sur le plan de la qualité.

2 Formation continue et droits acquis; BMS no 18/2006.

3 Commission paritaire «Bases de données de la valeur intrinsèque et des unités fonctionnelles réunissant les assureurs-maladie, représentés par santésuisse, les assureurs-accidents, représentés par le Service central des tarifs médicaux LAA (SCTM) ainsi que les fournisseurs de prestations, représentés par la FMH et $\mathrm{H}+$.
Depuis l'entrée en vigueur du TARMED au $1^{\text {er }}$ mai 2003 pour l'assurance-accidents, et au $1^{\text {er }}$ janvier 2004 pour l'assurance-maladie, les médecins ne peuvent en principe facturer la plupart des positions tarifaires que lorsqu'ils disposent de la «valeur intrinsèque» correspondante. La base contractuelle de ce mode de faire se trouve dans le concept de valeur intrinsèque version 9.0 datant de 2001. Ce concept a été approuvé par les membres de la FMH dans le cadre d'une votation générale, en même temps que l'ouvrage tarifaire TARMED.

Par «valeur intrinsèque», on entend les titres de spécialiste, formations approfondies et attestations de formation complémentaire tels qu'ils sont réglés dans la Réglementation pour la formation postgraduée. Pour que les médecins puissent continuer à exercer leur profession dans le même cadre qu'avant le TARMED, le concept de valeur intrinsèque contient la notion de garantie des droits acquis. Tout médecin peut ainsi continuer de facturer des prestations qu'il a fournies sous sa propre responsabilité, régulièrement et sans contestation sur le plan de la qualité, pendant les trois ans qui ont précédé l'entrée en vigueur du TARMED, même s'il ou elle ne dispose pas des titres ou certificats exigés pour cela. ${ }^{1}$ Ces droits acquis ne restent toutefois préservés que si le médecin accomplit une formation continue séparée pour les prestations concernées dans les trois ans suivant l'introduction du TARMED. ${ }^{2} \mathrm{La}$ première période de trois ans prévue pour l'attestation de cette formation continue est arrivée à échéance le 31 décembre 2006.

\section{Une banque de données créée par la FMH en trois mois}

Suite à un ultimatum fixé en juin 2004 par la commission $\mathrm{PaKoDig}^{3}$, la FMH a réussi le tour de force de mettre au point en trois mois une banque de données intégrée dans son logiciel de gestion et qui répondait aux conditions fixées par contrat pour une mise en service par les assureurs. Depuis lors, la banque de données de la valeur intrinsèque est complétée et mise à jour en permanence par la FMH, laquelle informe mensuellement la PaKoDig de son développement et de son état du moment. La FMH a ainsi appliqué jusqu'à présent la convention conclue. Cette per-

\section{Banque de données de la valeur intrinsèque - une histoire sans fin}

$2001 / 2002$

- L'ensemble des conventions TARMED est finalisé et entre en vigueur.

- La base contractuelle de la banque de données de la valeur intrinsèque est fixée dans le Concept de valeur intrinsèque 9.0, lequel fait partie intégrante des conventions TARMED.

\section{2}

Les membres de la FMH approuvent le TARMED et les conventions qui s'y rapportent dans le cadre d'une votation générale.

\section{3}

- La FMH effectue le recensement de la valeur intrinsèque auprès de ses membres et des non-membres connus à cette époque.

- Les données sont saisies sur un serveur externe à la FMH.

\section{Juin 2004}

- La FMH a du retard dans la mise en œuvre de la convention.

- Les assureurs siégeant à la commission PaKoDig fixent un ultimatum avec effet au 30 septembre 2004, en vue de reprendre l'ensemble de la banque de données de la valeur intrinsèque.

\section{Juillet 2004}

- Le Comité central nouvellement élu et le groupe de projet qu'il instaure reprennent le projet relatif à la valeur intrinsèque. Ils créent les conditions nécessaires à la mise en service de la banque de données de la valeur intrinsèque.

- Les données de valeur intrinsèque à disposition sont transférées dans une banque de données et intégrées dans le logiciel de gestion interne de la FMH.

\section{Septembre 2004}

- La banque de données de la valeur intrinsèque est prête à fonctionner, les conditions des assureurs sont remplies. Lors d'une $\rightarrow$ 
sévérance et ce respect des engagements pris n'ont de loin pas recueilli l'adhésion de tous les membres de la FMH. Nombreux sont ceux qui ne voient pas le sens de telles démarches et estiment qu'elles sont une perte de temps. Une réaction tout à fait compréhensible si l'on songe que les assureurs n'ont cessé d'annoncer leur intention de mettre en service cette banque de données, mais ne l'ont jamais fait à ce jour.

\section{myFMH permet aux membres de gérer eux-mêmes leurs valeurs intrinsèques}

Les tâches administratives de saisie, de gestion et de mise à jour des données de valeur intrinsèque sont complexes et prennent du temps, aussi bien pour la FMH que pour ses membres. La plateforme Internet myFMH créée en 2006 permet de simplifier ce processus. Les membres FMH peuvent à tout moment accéder à leur profil personnel de valeurs intrinsèques, compléter ou biffer leurs positions de droits acquis, valider leur nouveau profil et attester la formation continue qu'ils ont accomplie. Leur attestation de valeur intrinsèque est ainsi toujours disponible dans sa version la plus récente et ils peuvent la consulter et l'imprimer comme ils le désirent.

L'application «Mon profil de valeurs intrinsèques», qui contient des données personnelles spécifiques très sensibles, est sécurisée en conséquence. Les membres FMH qui possèdent un abonnement au réseau HIN (Health Info Net) bénéficient d'un haut degré de protection grâce à la technologie de sécurité ASAS. Une protection équivalente est offerte à tous les autres membres, moyennant l'introduction d'un mot de passe personnel et d'un code exigé par le système et provenant d'une liste de codes de sécurité.

Quiconque désire accéder pour la première fois à la plate-forme Internet $m y \mathrm{FMH}$ doit s'enregistrer. Les membres FMH en exercice ont tous reçu les documents d'enregistrement nécessaires par courrier postal entre juin et novembre 2006. Ceux-ci sont également envoyés aux personnes qui ont adhéré à la FMH depuis lors. En cas de difficultés lors de l'enregistrement, une ligne d'assistance est à disposition au numéro de tél. 0313591259 (du lundi au vendredi de 8 à 12 et de 13 à 17 heures) et à l'adresse électronique info-dig@fmh.ch.

\section{Non-remboursement de prestations en I'absence de la valeur intrinsèque exigée} Parmi les quelque 18000 membres FMH qui disposent de positions relevant des droits acquis, près de 11000 s'étaient enregistrés dans $m y \mathrm{FMH}$ jusqu'à la fin de la première période de trois ans, plus de 9000 y avaient actualisé et validé leurs visite au secrétariat général de la FMH, les délégués de la PaKoDig peuvent se convaincre qu'il est possible de consulter les données de valeur intrinsèque en respectant la convention.

- L'ultimatum est fixé à fin 2004 puis il est annulé.

- Les assureurs refusent de mettre en service la banque de données de la valeur intrinsèque.

- Les assureurs justifient leur refus en invoquant des questions techniques et continuent de demander la pleine possession de la banque de données. La FMH rejette cette demande en rappelant les bases contractuelles en vigueur ainsi que les engagements pris.

\section{Février/mars 2005}

- Le SCTM (Service central des tarifs médicaux $\mathrm{AA} / \mathrm{AM} / \mathrm{AI})$ teste avec succès la consultation en ligne des données de valeur intrinsèque.

- Dans son rapport, le SCTM constate que les services en ligne de la FMH fonctionnent sur le plan technique lorsqu'on utilise un client ASAS ainsi que les applications Java concernées sur un ordinateur isolé raccordé à l'Internet.

- Des solutions sont cherchées en commun.

\section{Mars 2005}

Le Comité de direction de TARMED Suisse opte pour une solution transitoire. Il décide que les prestations correspondant aux titres de spécialiste et aux attestations de formation complémentaire seront livrées sous la forme d'une banque de données pour l'ensemble des médecins établissant des factures. Il précise que les prestations relevant des droits acquis et les éventuelles prestations à fortes exigences seront communiquées sur demande en présence de factures concrètes, avec possibilité pour l'assureur de compléter ses données locales.

\section{Mai 2005}

La PaKoDig désigne deux groupes de travail chargés des aspects techniques et contractuels de la mise en œuvre de la solution transitoire.

\section{Octobre 2005}

Les deux groupes de travail ont terminé leurs travaux et remettent leurs rapports à la PaKoDig.

\section{Novembre 2005}

- Lors de sa séance, la PaKoDig note que le groupe de travail a achevé son mandat dans le délai imparti et qu'il demande l'approbation de la PaKoDig et du Comité de $\rightarrow$ 
données de valeur intrinsèque et presque 8500 $\mathrm{y}$ avaient attesté leur formation continue.

Lors de la mise à jour des profils personnels de valeur intrinsèque, de très nombreuses positions de droits acquis ont été biffées. Si l'on s'attendait à cette tendance, son ampleur a toutefois surpris: non moins de 314000 positions de droits acquis ont été supprimées, et la banque de données en contient actuellement encore environ un million et demi.

Le graphique «Chiffres-clés de la banque de données de la valeur intrinsèque» montre l'état actuel de la banque (août 2007).

Après avoir connu une augmentation marquée vers la fin 2006 - délai fixé pour déclarer la formation continue effectuée pour les positions de droits acquis - les enregistrements, validations et déclarations de formation continue ont nettement diminué. On constate un désintérêt croissant à cet égard. Toutefois, tant que les contrats correspondants sont en vigueur, le personnel chargé de gérer la banque de données de la valeur intrinsèque est tenu de protéger les membres FMH de surprises désagréables telles que le non-remboursement de prestations facturées en cas de valeur intrinsèque manquante, et d'attirer leur attention sur les conséquences juridiques de déclarations inexactes. Une assistance concrète en cas d'exigences injustifiées - ou de tentatives éventuelles de contourner les bases contractuelles -, de la part de certains assureurs, fait partie de ce service. direction pour poursuivre son activité. La PaKoDig précise que la validation des factures pourra débuter sur cette base dans les prochaines semaines, une fois que les assureurs auront donné leur approbation.

- Compte tenu des propositions qui lui ont été transmises par la PaKoDig, le Comité de direction décide que celle-ci peut poursuivre ses travaux. Les règlements doivent être allégés et examinés par des juristes. On est d'accord que la FMH, H+ et la CTM (Commission des tarifs médicaux) appliquent la solution transitoire.

- santésuisse émet des réserves quant à la réalisation de la solution transitoire dans le domaine de la LAMal.

\section{Mai 2006}

- santésuisse déclare clairement ne pas vouloir mettre en service la banque de données de la valeur intrinsèque.

- La FMH et la CTM souhaitent résoudre la question de façon bilatérale. Une proposition est élaborée et mise en consultation auprès des partenaires.

- Des informations communiquées par des membres de la FMH permettent de supposer que certains assureurs-maladie cherchent à contourner les bases contractuelles: ceux-ci menacent de ne pas payer les prestations facturées si le médecin concerné n'envoie $\rightarrow$

Figure 1

Chiffres-clés de la banque de données de la valeur intrinsèque.

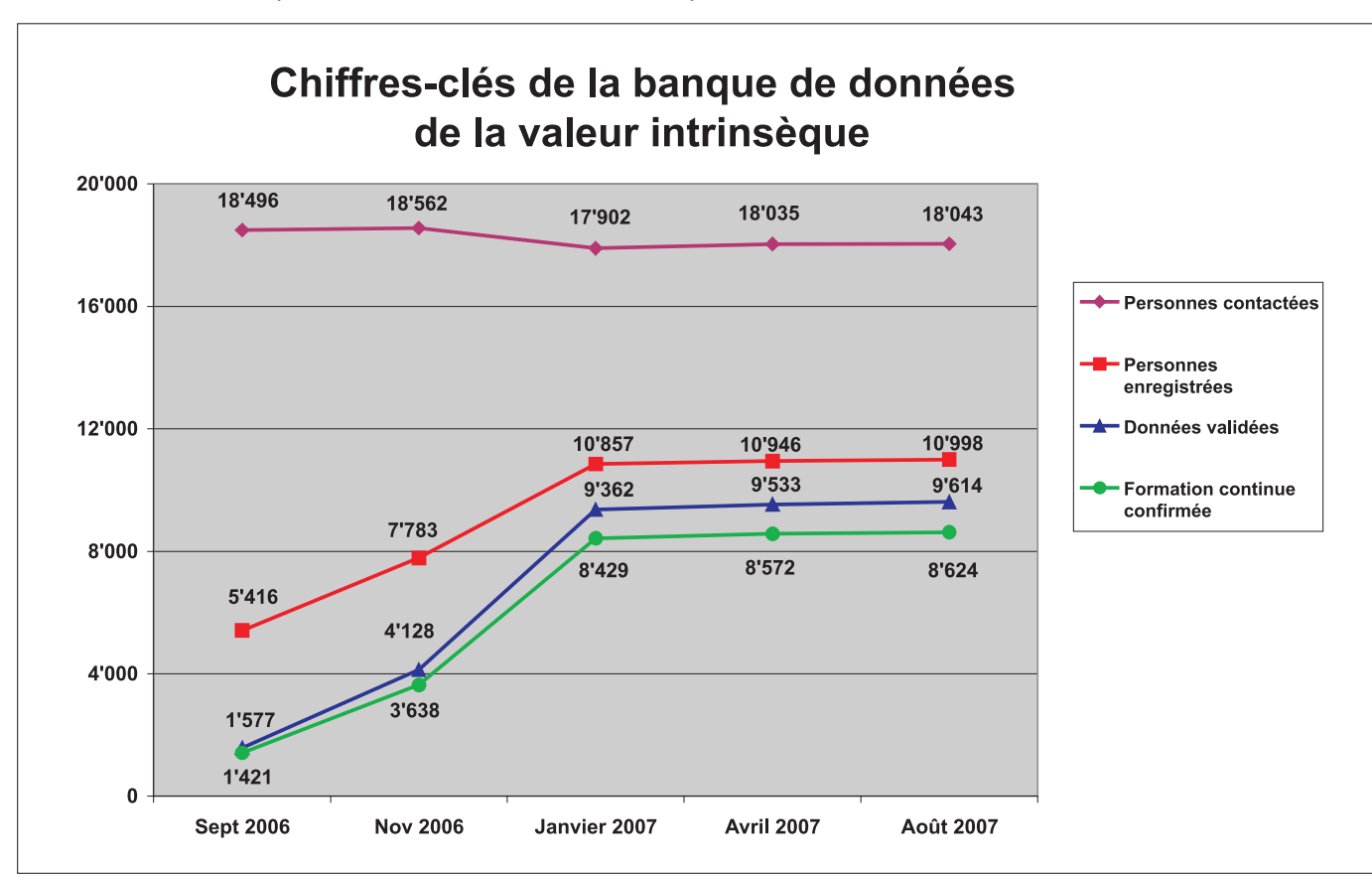


Vérification de la valeur intrinsèque dans le domaine AA/AM/Al: situation actuelle

Fin mars 2007, les organes compétents de la FMH et de la CTM ont décidé de mettre en service la banque de données de la valeur intrinsèque pour la facturation dans les domaines de l'assurance-accidents, de l'assurance-invalidité et de I'assurance militaire. Le calendrier suivant a été fixé:

- $1^{\text {er }}$ juin 2007: phase de tests techniques;

- $1^{\text {er }}$ octobre 2007: mise en service par étapes en vue d'une exploitation régulière, avec conséquences sur le processus de facturation;

- 1 $^{\text {er }}$ janvier 2008: entrée en fonction définitive de la banque de données.

Qu'est-ce que cela signifie pour les médecins qui ont indiqué des prestations sous les droits acquis dans leur profil de valeur intrinsèque?

Tout médecin qui facture des prestations à la charge des assurance-accidents, invalidité et militaire doit faire valider son profil de valeur intrinsèque et accomplir une formation continue séparée. Si, au moment de l'évaluation des valeurs intrinsèques par la CTM, la validation et l'attestation de la formation continue accomplie font défaut, les assureurs-accidents ont le droit de refuser de rembourser ces prestations.

Vous pouvez vérifier et valider simplement et rapidement votre profil personnel de valeur intrinsèque sur myFMH (www.myfmh.ch).

Ligne d'assistance: $\mathrm{n}^{\circ}$ de tél. 0313591259 (du lundi au vendredi de 8 à 12 et de 13 à 17 heures) et adresse électronique info-dig@fmh.ch

\section{Vérification ou non des valeurs intrinsèque? Un coup d'œil vers l'avenir}

Conformément au contrat signé, une banque des données de la valeur intrinsèque est à disposition auprès de la FMH depuis fin septembre 2004. Vu que les assureurs (santésuisse et Commission des tarifs médicaux LAA), pour leur part, n'ont pas voulu faire usage de cette banque selon ce qui avait été convenu contractuellement ni n'ont fait part, jusqu'à il y a six mois, de leur intention de l'utiliser dans un proche avenir, le Comité de direction de TARMED Suisse a pris la décision suivante à fin 2006 :

«Mise en service de la banque de données de la valeur intrinsèque: le Comité de direction de TARMED Suisse a décidé de donner la priorité au projet de sremaniement de la valeur intrinsèque qualitative et quantitative), qui exigera des travaux très importants, et de repousser à plus tard la mise en service de la banque de données de la valeur intrinsèque. Cela signifie que les assureurs ne pourront pas, jusqu'à nouvel avis, vérifier les valeurs intrinsèques des médecins. En cas de soupçon sérieux de facturation abusive en lien avec la valeur intrinsèque de tel ou tel praticien, celle-ci pourra être contrôlée de manière individuelle moyennant une demande adressée à la commission PaKoDig. Cette dernière prie les organisations concernées de communiquer cette nouvelle à leurs membres de la manière qui leur semblera appropriée.»

Fin mars 2007, la $\mathrm{CTM}^{4}$ a décidé de reprendre les négociations bilatérales en vue d'une mise en service de la banque de données. Les travaux pas son attestation de valeur intrinsèque; dans certains cas, ils adressent cette injonction simultanément au médecin et à son patient!

\section{Septembre à décembre 2006}

La CTM ne s'exprime plus sur la proposition précitée. Il apparaît qu'ici également, la mise en service n'aura pas lieu.

\section{Décembre 2006}

- A la séance de la PaKoDig, santésuisse exige une solution définitive, c'est-à-dire l'ouverture de toute la banque de données dès le $1^{\text {er }}$ janvier 2007.

- A ce jour, santésuisse n'a pas encore établi la liste des conditions correspondantes.

\section{Janvier 2007}

La PaKoDig ainsi que le Comité de direction de TARMED Suisse constatent que la solution définitive souhaitée par santésuisse ne correspond plus aux conditions contractuelles et qu'elle ne peut donc pas être appliquée pour l'instant. La non-mise en service de la banque de données de la valeur intrinsèque est formellement décidée et communiquée aux partenaires.

\section{Janvier à mars 2007}

Tant que la suite du processus ne sera pas clarifiée, personne n'est prêt à entreprendre de nouveaux travaux. La solution transitoire ne donne lieu à aucune dissension fondamentale entre la FMH et la CTM. La CTM signale qu'elle va mettre en service la consultation en ligne testée en février et mars 2005. La CTM, H+ et la FMH reprennent leurs travaux. Un calendrier est fixé (cf. encadré).

\section{Juillet 2007}

La phase de tests techniques est en cours depuis début juin. Jusqu'à présent, le SCTM a réalisé des tests de fonctionnement.

correspondants ont immédiatement été mis en route. (cf. encadré).

santésuisse, quant à elle, n'a jusqu'ici fait part d'aucune intention officielle de procéder à une telle mise en service. L'avenir reste ouvert. Comme évoqué ci-avant, TARMED Suisse a décidé de lancer pendant l'année en cours un projet de révision de la valeur intrinsèque qualitative et quantitative, dont l'exécution a par ailleurs déjà pris du retard par rapport au calendrier prévu. 